\title{
VIOLENCIA HOSPITALARIA EN PACIENTES
}

\author{
HOSPITAL'S VIOLENCE IN PATIENTS
}

\author{
MÓNICA BURGOS MORENO* y TATIANA PARAVIC KLIJN**
}

\begin{abstract}
RESUMEN
El paciente/usuario se presenta susceptible a todos los estímulos y relaciones que surgen en el contexto hospitalario, en una atención que se reconoce como despersonalizada y falta de privacidad, lo que puede convertirle en una potencial víctima de actos violentos. Por la importancia que reviste otorgar una atención en salud basada en el respeto y la dignidad hacia los usuarios, se hace necesario estudiar el fenómeno de la violencia que desde la óptica de los pacientes pudiera encontrarse en los centros de salud; con la finalidad de contribuir al mejoramiento de la atención y cuidado que le es otorgado en los centros hospitarios.
\end{abstract}

Palabras claves: Violencia hospitalaria, violencia en pacientes.

\begin{abstract}
Patients/users appear susceptible to all kinds of stimuli and relationship arising within the hospital context where the care given is defined as impersonal and lacking privacy, posing the potential threat of becoming a victim to violent actions. Aiming at making a contribution to the improvement of health care given in hospitals, the importance of providing health care based on dignity and respect for the patients calls for a study of the violence issue that patients seem to perceive within health care contexts.
\end{abstract}

Keywords: Hospital's violence, violence in patients.

Recepcionado: 31.04.2003. Aceptado: 4.06.2003.

\section{INTRODUCCIÓN}

La violencia, tan antigua como el hombre mismo (Domenach, 1981), es un tema prioritario de investigación debido al considerable aumento que ha presentado en los últimos decenios, atravesando fronteras, edad, raza, condición socioeconómica, religión y sexo (Consejo Internacional de Enfermería, 1999), además de las diversas consecuencias que tiene en los individuos, familias y comunidad en general, efectos también reconocidos en el ámbito de la salud de las personas, lo que ha llevado a considerar este fenómeno como una epidemia (OMS, 1996).

Así la violencia, con sus negativos efectos $y$ presente en todo lugar en donde el queha- cer humano se manifiesta, ha motivado también estudios sobre esta problemática en los lugares de trabajo, en los que se reconoce que los ambientes laborales más expuestos a actos violentos son precisamente los de atención sanitaria, en especial los trabajadores que se desempeñan en establecimientos hospitalarios. Sin embargo, este tema no ha sido abordado igualmente desde el prisma de quienes reciben la atención en salud, con lo cual existe un vacío en el conocimiento sobre la violencia percibida por los pacientes 0 usuarios de los servicios hospitalarios en nuestro medio.

Estimando que el paciente es el principal sujeto de atención, que su condición de enfermo en un ambiente ajeno -muchas veces

\footnotetext{
*Enfermera. Pabellones Quirúrgicos, Unidad Recuperación Anestésica, Hospital Las Higueras de Talcahuano. Magister en Enfermería, Universidad de Concepción, Chile.

${ }^{* *}$ Profesor Titular Departamento de Enfermería, Facultad de Medicina, Universidad de Concepción. Magister en Enfermería, mención Salud Comunitaria, Universidad de Concepción, Chile. Doctora en Enfermería, U.SP. Brazil
} 
desconocido-, rodeado de innumerables estímulos y dependiente en grados variables del cuidado de otros, hace que se presente vulnerable a los diversos comportamientos violentos o a ser una potencial víctima de ellos, obliga a estudiar este fenómeno de la violencia en los contextos de atención en salud desde la óptica de los pacientes.

\section{VIOLENCIA Y EL CONTEXTO DE SALUD}

Se entiende por violencia cualquier comportamiento físico, psicológico/emocional y/o sexual que, por medio de la acción u omisión, provoca daño a las personas (Puget y Berenstain, 1998). La violencia, así entendi$\mathrm{da}$, se comprueba en hechos cotidianos a los cuales el individuo está expuesto (víctima) o es el responsable (victimario). Hechos en que la violencia puede estar dirigida hacia sí mismo, hacia las personas o hacia las cosas, y que puede ser directa o implícita (Rapaport, 1992). En general, cualquiera sea su dirección, tenemos la tendencia a identificarla de inmediato con ciertas acciones concretas: golpes, balazos y, en su expresión más elocuente y descarnada, la guerra. Es decir, se relaciona con hechos visibles y manifiestos de la agresión física que provoca daños que pueden llegar a producir la muerte (Hacker, 1973). Se reconoce también como expresión de violencia aquellas situaciones en las que existe una percepción de mala calidad de servicios o de mala atención, no satisfacción de necesidades de un cliente, proveer de servicios que están muy lejos de las expectativas del usuario, cuando se abusa o se recibe un trato injusto (Chapell y Di Martino, 1998).

La violencia identificada como fenómeno multicausal (Chapell y Di Martino, 1998; Buinic et al., 1999; Programa de la Mujer, Salud y Desarrollo, 1999 y OMS, 2002), ha sido abordada por diversos estudios desarrollados por la OIT (1988) como problemática en los ambientes de trabajo, concluyendo que algunos ambientes laborales están más expuestos que otros a diferentes tipos de comportamientos violentos.
Entre los trabajadores con mayor riesgo de actos violentos, según los estudios, se encuentran los que se desempeñan en el sector salud, especialmente en el área intrahospitalaria (Mahoney, 1990; Nogareda, 1990; Beck y col., 1992; Aranetz, 1994 y Sánchez, 2002). Para el CIE (2002) esta problemática tiene carácter universal, es decir, afecta a todos los trabajadores de atención sanitaria en mayor o menor grado, principalmente al personal de ambulancias, médicos y personal de enfermería que se desempeña en servicios hospitalarios. Esta violencia de los funcionarios en el lugar de trabajo también puede generar en ellos comportamientos violentos, extendiéndose hacia los usuarios de los servicios de salud (Kignma, 1999), los que representan el principal foco de atención en este contexto.

\section{PACIENTES EN EL CONTEXTO HOSPITALARIO}

Las personas deben cumplir normalmente con numerosas tareas y compromisos en las distintas etapas de la vida, colegio, trabajo, familia, etc., que aseguren su desarrollo y desenvolvimiento normal en la sociedad; sin embargo esto puede verse alterado por la condición de enfermedad y más aún cuando es necesario el ingreso a un hospital. Esta situación hace al individuo vulnerable a variados estímulos, condiciones e interrelaciones que se desarrollan en este medio en el que adopta o pasa ser incluido en el "status de paciente" (Martínez, 1997). En efecto, el paciente se enfrenta a un medio con estímulos y situaciones diversas que no solamente alteran la satisfacción de necesidades de orden elemental o biológicas, como pueden ser el dormir o el alimentarse, sino también aquéllas de orden superior, especialmente las que comprometen su esfera psico-afectiva. En este punto Jiménez (2000) señala que en el paciente hospitalizado no solamente ve afectado el aspecto económico, el funcionamiento familiar y las responsabilidades previamente adquiridas, sino también se altera su 
autoestima y se producen cambios emocionales intensos que requieren de reajustes.

Estos cambios, según Moro (1999), repercuten además en las necesidades de seguridad y de pertenencia, las que se encuentran afectadas en el individuo aquejado por alguna enfermedad. Esta alteración que sufre toda persona enferma se acentúa especialmente cuando el individuo requiere la internación en un establecimiento de salud, ya que la separación del ambiente familiar, conocido y estable por otro ambiente extraño, con una serie de reglamentación interna, relación con múltiples personas y a veces falto de privacidad, potencia los sentimientos de inferioridad y reducción que pueden surgir en las personas. Este autor señala que la persona enferma erradicada de su territorio vital a otro generalmente desconocido pierde en alguna medida la imagen de sí, aparecen sentimientos de culpa y vergüenza, que aumentan con el tratamiento despersonalizado de los procesos diagnósticos, terapéuticos y asistenciales a los que se ve sometido durante su hospitalización. Entonces las repercusiones de la enfermedad se hacen mucho más evidentes cuando, por su gravedad o por las condiciones socioeconómicas del paciente, imponen la necesidad de hospitalización.

El hospital, junto con ofrecer atención sanitaria, se constituye entonces en un sistema social complejo y delicado, en el cual la presencia de multiplicidad de personas con distintos roles, tales como profesionales, técnicos, pacientes, alumnos, familiares, entre otros, conforman una red interactiva que puede inducir a desarrollar o modificar actitudes (Valenzuela, 1995) que pueden llevar a hechos agresivos o violentos. Entonces la hospitalización puede tener una condición de vulnerabilidad aportada por la enfermedad, que puede ser la causa de la pérdida del sentido del control físico y/o psicológico y por el significado de someterse a un ambiente complejo y muchas veces desconocido.

Es conveniente reflexionar sobre algunos aspectos anteriormente citados, sobre la vivencia de este ser humano, paciente ingresado en un hospital, aspectos que pueden ser considerados inapropiados o en alguna medida violentadores durante la atención que el equipo de salud brinda a los pacientes y que, asociado a la masificación y complejidad creciente de la atención en salud, no sean analizados con la frecuencia y profundidad que merecen. Según la experiencia de las autoras de este artículo, existe ciertamente una dualidad en la satisfacción de la necesidad de seguridad que experimenta el paciente hospitalizado, ya que, por un lado, el hospital representa el control y / o vigilancia del problema de salud que le aqueja en cuanto a cuidados y evaluación, pero, por otro, se acentúan también los sentimientos de inseguridad e inestabilidad emocional, relacionados con la separación familiar y el someterse a normas y reglamentación internas que rigen a cada establecimiento de salud. Un paciente recién ingresado desconoce esta reglamentación y si no es dada a conocer, explicada oportuna y claramente, puede representar una amenaza para el paciente y su familia, como por ejemplo el desalojo de las salas de la visita de familiares cuando ya se ha cumplido el horario, impedir el ingreso de la familia en áreas de acceso restringido, negar el uso de sus propias ropas para ser remplazada por una bata de hospital, entre otras.

Otro aspecto que pareciera ser inherente a la hospitalización es la falta de intimidad, definida como toda aquella realidad oculta, relativa a un sujeto o grupo determinado que merece reserva (Vacarezza, 2000); ésta se ve de alguna manera vulnerada en el paciente hospitalizado. La falta de respeto a la privacidad se manifiesta desde la invasión no sólo a espacio territorial, reducido a una cama y un velador, sino también al ser examinado en la cama de hospital por innumerables personas a las que generalmente desconoce y respondiendo variadas preguntas relativas a su historia de vida personal, muchas veces sin una explicación previa del porqué son necesarios estos procedimientos y estas respuestas. Pareciera ser más importante el órgano afectado que la persona enferma, olvidando el respeto a la desnudez y el pudor del otro (Rodríguez, 1999). Esto se ha ido presentando en la 
medida en que la enfermedad monopoliza la atención e inconscientemente se olvida la naturaleza humana de la atención de salud (Iceta, 1996), tornándose ésta fría e impersonal, lo que ha violentado la relación amistosa y de confianza, pasando más bien a un vínculo despersonalizado (Goic, 2000).

Esta despersonalización creciente, en la que la persona ha pasado a un lamentable segundo plano -ya sea por el aumento de la demanda asistencial y / o tecnificación de la atención de salud que otorgan los hospitales e instituciones afines, donde la actitud humanizante que debe caracterizar la relación entre prestadores y usuarios se ha ido deteriorando-, ha reducido la visión integral del paciente como ser biopsicosocial, adjudicándose el protagonismo a la enfermedad (Iceta, 1996). Esta visión, que actualmente se presenta parcelada, se manifiesta en hechos tan cotidianos que casi son parte de la "normalidad" en las relaciones que se establecen entre los prestadores y los usuarios; en un apremio de tiempo, tal vez por simplificar algún aspecto dentro de este ambiente tan complejo, se va perdiendo algo tan esencial y propio de cada ser humano como es la identidad, al reconocerle según la enfermedad que le aqueja (el de la hernia, el tec), el órgano afectado (el de la vesícula, la del pulmón), el examen solicitado (la de la radiografía, el del escáner), la intervención realizada (el del bypass, el del transplante) y por la sala de hospitalización (el del 20-9, la de la 33-C), y no por su nombre.

En este punto, Henderson (1988) señala que existen otros signos o situaciones en que las relaciones con el equipo de salud dejan de manifiesto el protagonismo de la enfermedad -y en las que se exponen claramente a los pacientes/usuarios a un segundo plano y que los lleva a sentirse por lo menos incómodos o molestos-, tales como el tutear a los adultos, dirigirse hacia otra persona sin identificarse previamente, hacer comentarios en presencia de otro paciente, realizar procedimientos sin el consentimiento, trasladar a los pacientes de sala sin explicación alguna. A estas situaciones podrían agregarse: tratar de hija/o o abuela/o a quienes no lo son nuestros, el entrar a una sala sin saludar, retirarse sin despedirse, usar terminología técnica que imposibilita la comprensión del paciente, tratar de exagerado el dolor del otro.

Existen probablemente otros hechos que no sólo deterioran la relación entre prestadores y usuarios en el hospital, sino que también vulneran los derechos de éstos últimos, los que, inadvertidamente y en alguna medida, forman parte de la cotidianidad en la atención de salud, reconocida como asimétrica y jerárquica entre cuidadores y pacientes de hospitales, especialmente en los establecimientos públicos (Rocha y cols., 2000). Estos hechos pueden convertir al paciente en una víctima de actos considerados agresivos o violentos, debido a una atención despersonalizada y falta de privacidad, en la que impera la enfermedad y la técnica, y no el paciente como sujeto principal de la atención. Las profesiones del área de la salud que aspiran el bienestar del individuo deben otorgar una atención integral basada en la dignidad inalienable e inherente de cada persona que se encuentre hospitalizada.

Esta atención basada en el respeto es un punto importante del código deontológico del CIE (2000) para la profesión de enfermería. Contempla en uno de sus cuatro elementos que regulan la conducta ética, la enfermería y las personas que, al dispensar cuidados, la enfermera promoverá un entorno en el que se respeten los derechos humanos, valores, costumbres y creencias de los individuos. El respeto al otro ser humano paciente y los derechos y deberes que como tal tiene deben, por tanto, condicionar todas las acciones de enfermería. El respeto es un principio que rige desde su concepción hasta el suspiro final y aún más allá de su cuerpo y después de la muerte, y que no debe ser postergado por los avances tecnológicos (Arroyo , 2000).

Para el profesional de enfermería no solamente importa administrar hábilmente los diversos procedimientos que exige el quehacer diario, sino estar atentos a las distintas manifestaciones de los pacientes, derivadas de la propia enfermedad, tratamientos y tam- 
bién de todos aquellos provenientes del medio hospitalario y de relación que surgen en la atención brindada por el equipo de salud a los usuarios, relación que en los últimos tiempos se ha deteriorado, dando paso a conductas y situaciones que pueden ser consideradas como violentas por los pacientes, por cuanto se pierde el principio fundamental del respeto, y que en mayor o menor grado pueden alterar su bienestar. Esta situación en la que los usuarios podrían considerarse víctimas potenciales de diferentes tipos de manifestaciones violentas, que van desde no tratarlo por su nombre o no cumplir con las expectativas de la atención prestada, merece atención por la vulnerabilidad que éstos presentan y las características de su condición, por la importancia que reviste otorgar una buena atención de salud a los pacientes, por la violencia reconocida en todos los contextos sociales, incluidos los servicios de salud, y por las escasas investigaciones en este tema desde la perspectiva de los pacientes. Se hace necesario, entonces, realizar un diagnóstico de la situación de violencia percibida por los pacientes en los diversos contextos de atención de salud y abordar esta problemática basándose en los resultados obtenidos, para proponer estrategias a objeto de prevenir y / o intervenir si la situación así lo requiere, contribuyendo de esta forma con el compromiso adquirido por el CIE (2001) de erradicar la violencia en las instituciones de salud, otorgando prestaciones sanitarias en centros de salud seguros y libres del flagelo de la violencia.

\section{REFERENCIAS BIBLIOGRÁFICAS}

ARNETZ, L. (1994). In: Chapell, D. y Di Martino, V. Violence at Work (pp. 73) Génova: International Labour Office.

ARROYO, G. (2000). Humanismo en enfermería. Revista Enferm IMSS, 8 (2), 61-63.

BECK, E. et al. (1992). In: Chapell, D. y Di Martino, V. Violence at Work (pp. 73). Génova: International Labour Office.

BUINIC, M., MORRISON, A. y SHIFTER, M. (1999). La violencia en América Latina y el Caribe: un marco de referencia para la acción. Extraído en octubre
15, 2002, disponible en http:/ / www.iadb.org/sds / publication/publication_515_s.htm

CHAPELL, D. \& DI MARTINO, V. (1998). Violence at Work (pp. 62-69). Génova: International Labour Office.

CONSEJO INTERNACIONAL DE ENFERMERÍA (1999). La violencia. Epidemia mundial. Boletín del CIE. Extraído el 11 septiembre 2000, disponible en http:/ / www.icn.ch/matters_violencesp.htm

CONSEJO INTERNACIONAL DE ENFERMERÍA (2001). Unidas frente a la violencia: Las enfermeras, dispuestas siempre a ayudarte. Extraído el 28 julio 2002, disponible en http:/ / www.icn.ch/

CONSEJO INTERNACIONAL DE ENFERMERÍA (2000). Código Deontológico del CIE para la profesión de enfermería. Extraído el 12 agosto 2002, disponible en http: / / www.icn.ch/icncodesp.pdf

CONSEJO INTERNACIONAL DE ENFERMERÍA (2002). Nuevos estudios muestran que la violencia en el lugar de trabajo es una amenaza para los servicios de salud en todo el mundo. Comunicado de prensa CIE. Extraído el 26 septiembre 2002, disponible en http:// www.icn.ch/violencia/ pr10_02sp.htm

GOIC, A. (2000). Es hora de pensar en los derechos de los pacientes. Una introducción. Revista Médica de Chile, 128, 1371-1373.

HACKER, F. (1973). En: Hijar, M.; López, M. y Blanco, $\mathrm{J}$. La violencia y sus repercusiones en la salud; reflexiones teóricas y magnitud del problema en México. Extraído el 18 julio 2001, disponible en http:/ / www.insp.mx/ salud/39/396-9.html

HENDERSON, V. (1988). Podemos dar un papel estelar a los pacientes. En: Investigación y Educación en Enfermería, 8 (1), 125-128.

ICETA, M. (1996). Bioética ¿para qué? Revista Bioética y Ciencias de la Salud, 2 (2), 39-34.

KINGMA, M. (1999). El incremento de la violencia en el lugar de trabajo es una amenaza para la enfermería y la prestación de los cuidados de salud. En Comunicado de prensa CIE. Extraído el 28 julio 2002, disponible en http://www.icn.ch/ prviolence_99sp.htm

JIMÉNEZ, P. (2000). Cuidados de la autoestima del paciente hospitalizado. Revista Sociedad de Enfermería Geriátrica y Gerontológica, 2, 71-78.

MAHONEY, B. (1990). En: Directrices: Para hacer frente a la violencia en el lugar de trabajo, CIE. Extraído el 28 noviembre 2001, disponible en http:/ / www.icn.ch/

MARTÍNEZ, A. (1997). El profesional de enfermería en su entorno de trabajo. Humanización de la asistencia. En Manual de ética y legislación en enfermería (pp. 171-176). Madrid: Mosby/Doyma.

MORO, A. (1999). El enfermo. En Manuale di Pastorale Sanitaria (pp. 211-212). Roma: Camilliane.

NOGAREDA C. (1990). In: Chapell, D. y Di Martino, V. Violence at Work. Génova: International Labour Office.

ORGANIZACIÓN INTERNACIONAL DEL TRABAJO (1998). La violencia en el trabajo: un problema 
mundial. Extraído el 26 septiembre 2001, disponible en http:/ / www.ilo.org/public/spanish/ bureau/inf/pr/1998/30.htm

ORGANIZACION MUNDIAL DE LA SALUD (1996). La violencia epidemia mundial. Extraído el 26 septiembre 2001, disponible en http: / / www.who.int / violence

ORGANIZACIÓN MUNDIAL DE LA SALUD (2002). World report on violence and healt. Génova: OMS. PROGRAMA DE LA MUJER, SALUD Y DESARROLLO (HDW) (1999). Violencia contra las mujeres y las niñas: el marco conceptual que se está implementando en diez países. Extraído el 20 agosto 2002, disponible en http:/ / www.paho.org/ spanish/gov/ce/msd/doc093.pdf

PUGET, J. Y BERENSTEIN, F. (1988). En: Del Cioppo. Violencia, mujer y género. Extraído el 7 noviembre 2000, disponible en: http:/ / www.fumtadip. com.ar/violencia.htm/

RAPAPORT, E. (1992). Psicología de la violencia. Revista Universitaria, 36, 32-39.
ROCHA, W., ROCHTI, W. y BELLATO, R. (2000). En: Proyecto de un estudo diagnostico das condiçoes de atendimento aos direitos a pessoa internada en um serviço público hospitalar na cidade de CuiabaMG. Brazil.

RODRÍGUEZ, C. (1999). Calidad de la atención en salud y ley 100 de seguridad social. En Bioética y Justicia Sanitaria (pp. 147-182). Colombia: El Bosque.

SÁNCHEZ, R. (2002). Percepción de violencia que afecta a los profesionales de enfermería de un hospital de la octava región. Tesis para optar al grado de Magister en Enfermería, Departamento de Enfermería, Universidad de Concepción, Concepción, Chile.

VACAREZZA, R. (2000). De los derechos del paciente. Revista Médica de Chile, 128, 1380-1384.

VALENZUELA, S. (1995). Factores que influyen en la actitud de los estudiantes de enfermería hacia el cuidado de pacientes durante sus experiencias clínicas médico-quirúrgicas. Tesis para optar al grado de Magister en Enfermería, Departamento de Enfermería, Universidad de Concepción, Concepción, Chile. 